\title{
AN OVERVIEW OF THE TIME-DEPENDENT ROTATIONAL MODULATION OF SATURNIAN RADIO EMISSIONS
}

\author{
D. A Gurnett*
}

\begin{abstract}
The discovery of Saturn kilometric radiation (SKR) was made by the Voyager spacecraft over three decades ago. The Voyager observations showed that SKR had a well-defined clock-like amplitude modulation with a period of $10 \mathrm{hr} 39 \mathrm{~min} 24 \pm 7 \mathrm{sec}$. Since then much has changed. In 2000 radio observations by the Ulysses spacecraft showed that the SKR modulation period varied by as much as several minutes on time scales of years. This long-term variability was subsequently confirmed by radio measurements from the Cassini spacecraft, which was put in orbit around Saturn on 1 July 2004. We now know that there are three basic types of Saturnian radio emissions: SKR at frequencies from about 50 to $1,200 \mathrm{kHz}$; narrowband (NB) emissions in two frequency ranges, near $5 \mathrm{kHz}$ and near $20 \mathrm{kHz}$; and whistler-mode auroral hiss at frequencies from a few $\mathrm{Hz}$ to several $\mathrm{kHz}$. All of these radio emissions display long-term variations in their modulation periods of up to one percent or more on time scales of years, with smaller variations on shorter time scales. For several years prior to Saturn's recent equinox (August 2009) these radio emissions displayed two dominant periods of about 10.6 and 10.8 hours. The 10.6-hour period has been shown to be associated with SKR and auroral hiss originating from the northern auroral zone; and the 10.8-hour period has been associated with SKR and auroral hiss originating from the southern auroral zone. The narrowband emissions observed during this period have the same two periods as the SKR and auroral hiss, but do not have the corresponding north-south symmetry. As equinox approached the two periods converged and now appear to have crossed several months after equinox. A similar crossing of the two components may have occurred in the Ulysses measurements of SKR during the Saturn's equinox fourteen years ago. Possible models for explaining these complex long term variations will be discussed.
\end{abstract}

* Department of Physics and Astronomy, University of Iowa, Iowa City, IA 52242, USA 
